Flavio Pechansky 1 Silvia C. Halpern 1 Mauro Soibelman 2 Carla Bicca 1 Cláudia Maciel Szobot 1 Ana Flávia Barros da Silva Lima 1 Akemi Scarlet Shiba 1

\section{Limites e alternativas para a implementação de um programa para dependentes químicos em risco para infecção pelo HIV utilizando o conceito de Rede Social}

\author{
Limits and alternatives to the implementation of \\ a program for intravenous drug users at risk of HIV \\ infection using the Social Network Approach
}

\footnotetext{
1 Departamento de Psiquiatria e Medicina Legal Faculdade de Medicina, Universidade Federal do Rio Grande do Sul. Rua Ramiro Barcelos 2350, 4 o andar, sala $400 \mathrm{~N}$, Porto Alegre, RS 90035-003, Brasil. fpechans@conex.com.br 2 Rua Vieira de Castro 150, sala 102, Porto Alegre, RS 90040-320, Brasil.
}

\begin{abstract}
The authors describe the development of a preventive program focused on intravenous drug users at risk of HIV infection, using the Social Network Approach as the intervention model. The authors describe the project's steps in a large university hospital in southern Brazil, emphasizing the unique methods and techniques developed by the treatment staff. Problems encountered during the project development are discussed, aimed at identifying the reasons why the program only achieved partial success. The authors identify critical issues, such as the use of a new technique not previously tried in Brazil, difficulties in maintaining IV drug users in treatment, lack of infrastructure for walk-in treatment, and the challenge of motivating staff and patients to continue treatment. The authors conclude by listing suggestions aimed at facilitating the development of new projects based on the same conceptual model.
\end{abstract}

Key words HIV; Acquired Immunodeficiency Syndrome; Community Networks; Substance Abuse

Resumo Os autores descrevem as etapas de execução de um programa de ações preventivas para usuários de drogas sob risco de infecção pelo vírus da imunodeficiência humana (HIV), utilizando como paradigma de intervenção um modelo de abordagem de redes sociais. São descritos os passos de instalação do projeto dentro de um grande hospital universitário do sul do Brasil, com ênfase nos métodos e técnicas originais desenvolvidos pela equipe de atendimento. As dificuldades de implementação do projeto são discutidas ao longo das seções, buscando identificar os motivos pelos quais o programa obteve êxito apenas parcial. Os autores destacam alguns pontos críticos, tais como a utilização de uma técnica ainda não difundida em nosso meio, a dificuldade de reter em tratamento usuários de drogas injetáveis, a falta de estrutura para atendimentos imediatos, e as dificuldades em motivar equipes e pacientes para o tratamento. Ao final do texto, são feitas sugestões com o objetivo de facilitar o desenvolvimento de novos projetos que busquem utilizar o mesmo modelo conceitual.

Palavras-chave HIV; Síndrome da Imunodeficiência Adquirida; Redes Comunitárias; Abuso de Substâncias 


\section{Introdução}

No Brasil, as abordagens de cunho preventivo para usuários de drogas, em especial as injetáveis (UDI), ainda apresentam lacunas. É freqüente o UDI ser estigmatizado pelo seu problema, devido à associação entre abuso de drogas e a transmissão do HIV. Faz-se portanto necessária uma abordagem que considere o UDI não apenas em seus aspectos clínicos, envolvendo sua desintoxicação e cuidados terapêuticos iniciais, mas também sua progressiva readaptação às condições sociais de seu meio, reinserindo-o de forma produtiva na sociedade.

Os modelos de prevenção atuais nos obrigam a perceber que o UDI convive com companheiros de droga e parceiros sexuais que não são necessariamente seus familiares, ficando portanto de fora das abordagens convencionais propostas até então. Estudos recentes realizados por Mandell et al. (1994) demonstraram ser vantajosa a inclusão da rede social neste modelo. Latkin et al. (1995) utilizam-se do conceito de rede social para descrever os seis domínios que comporiam o que se chama de "rede pessoal de suporte" de um indivíduo: assistência material, socialização, interação íntima, assistência física, informação de saúde e reforço positivo. A abordagem de rede social parte do princípio que o UDI compartilha uma fração significativa de sua vida com seu grupo de iguais, e que intervenções orientadas e focais podem gerar resultados positivos nas relações sociais entre UDI (intra-redes) e nas relações da rede social com o meio que o cerca (interredes). Para fins deste projeto os autores consideraram como rede social o grupo que compartilha drogas ou que possui práticas sexuais em comum, buscando valorizar as relações intraredes. Segundo Pechansky et al. (1996), o uso diário de álcool e maconha nestes grupos de UDI é freqüente (46\%) além de cocaína por mais de uma via ( $83 \%$ aspiram ocasionalmente, $84 \%$ injetam). Quando usam a via endovenosa, estes indivíduos o fazem na maioria das vezes em grupos e se dizem francamente preocupados com a infecção ou disseminação do HIV para terceiros. É freqüente o ato de compartilhar equipamentos de injeção ou a droga em si, como também o ato de injetar-se com o cônjuge, parceiro sexual ou amigos. É portanto evidente que os UDI se expõem à infecção e/ou reinfecção pelo HIV, sendo que as redes sociais desempenham um papel fundamental na manutenção desse processo.

Por volta de 1991, a cidade de Porto Alegre tinha a quarta maior concentração de casos comunicados de AIDS no Brasil e a transmissão homossexual era responsável pela maioria dos casos, havendo uma modificação significativa neste quadro nos anos seguintes. É possível citar, por exemplo, o estudo de Santos et al. (1994), que descreviam através de um estudo longitudinal com 405 pacientes, a modificação do perfil de clientes que buscavam atendimento relativo à disseminação do HIV. Havia um aumento do número de mulheres infectadas pelo vírus, assim como uma percepção de que a transmissão por via heterossexual era progressivamente responsável pela maioria dos casos.

Porto alegre tem sido responsável por aproximadamente $50 \%$ do total de casos notificados de AIDS nos últimos anos, e se acrescentarmos a esta proporção os casos de outros municípios da área metropolitana, a proporção de casos pode chegar a 70\% (Barcellos, 1998). Portanto, na iminência da descoberta de focos específicos para a transmissão do HIV através de múltiplos vetores também em nosso meio, é de utilidade conhecer e abordar algumas das circunstâncias peculiares onde usuários injetáveis que se dizem francamente preocupados com a infecção pelo HIV (Pechansky et al., 1997) se encontram em situação grupal. O Programa POAVHIVE - Venha Enfrentar o HIV, foi desenvolvido dentro desta filosofia de trabalho. O presente artigo descreve este projeto, financiado pelo Ministério da Saúde e o United Nations Drug Control Program (UNDCP) e desenvolvido em parceria com o Departamento de Psiquiatria e Medicina Legal da Faculdade de Medicina (FAMED) da Universidade Federal do Rio Grande do Sul (UFRGS). Este artigo relata os passos de execução do projeto inicial e descreve seus componentes, equipe técnica, infra-estrutura, pacientes e modalidades de atendimento. Os acertos e falhas percebidos pelos autores são comentados ao longo do texto, com o objetivo de compartilhar criticamente a experiência e os resultados obtidos.

\section{Antecedentes do projeto}

Ao propor a execução do POAVIVHE, o Departamento de Psiquiatria e Medicina Legal da UFRGS iniciou uma série de projetos em colaboração com a Universidade de Delaware, a Universidade de Miami, e com a Universidade da Pennsylvania (Pechansky et al., 1994; Woody et al., 1995). Estes projetos permitiram documentar as características dos UDI que buscam atendimento em diversos locais de Porto Alegre, além de estimar seu risco de infecção pelo HIV, criando uma pequena estrutura para pesquisa e atendimento desta comunidade. Como 
parte integrante desta linha de atuação, o Projeto POAVHIVE se propõe a desenvolver e testar métodos de recuperação social para estes pacientes, incluindo tratamento psicoterápico em grupo com técnicas motivacionais, jogos terapêuticos, psicofarmacologia, intervenções psicossociais junto à rede do UDI e sua família, e a articulação do programa com recursos da comunidade.

\section{Principais objetivos}

Os objetivos principais do POAVHIVE, na sua concepção inicial, foram: (a) Identificar e registrar características de 36 UDI e suas redes sociais na cidade de Porto Alegre; (b) estabelecer características motivacionais e estágios de mudança no UDI e em sua rede social; (c) conhecer o impacto do uso de drogas nas principais áreas de vida do UDI e de sua rede: família, lei, status físico, status emocional, uso geral de drogas; (d) aplicar módulos terapêuticos e preventivos, desenvolvidos a partir das informações produzidas pelos próprios UDI, visando esclarecimento e mudanças de atitude com o objetivo de diminuir o uso de drogas em geral - particularmente o uso das injetáveis, e comportamentos sexuais de exposição a risco; (e) buscar identificar modelos de reinserção familiar e social do UDI em seu meio; (f) formar multiplicadores sociais e agentes de saúde treinados especificamente em técnicas grupais de atendimento e prevenção; (g) descrever os processos e resultados obtidos ao longo do projeto; (h) formar uma base de dados detalhada sobre características de uso de drogas e hábitos sociais de UDI e suas redes sociais na cidade de Porto Alegre; (i) desenvolver e centralizar contatos entre profissionais especialistas e supervisão sistemática do trabalho por experts do país, e discutir sistematicamente os achados do projeto com profissionais especialistas.

\section{Métodos e técnicas}

O tempo total de duração do projeto foi de 13 meses, com os três primeiros destinados à instalação do programa, e do terceiro ao quinto mês a previsão de ampla reforma do espaço físico onde o programa iria operar. Devido a atrasos no cronograma os atendimentos tiveram que ser realizados dentro do hospital, o que modificou a logística do projeto. Os pacientes encaminhados para o projeto seriam inicialmente acolhidos por uma enfermeira psiquiátrica, que explicaria o procedimento de entra- da no programa, sendo após submetidos a uma série de instrumentos de avaliação padronizados, com a finalidade de mensurar o impacto do uso de drogas, a relação do sujeito com elas em seus aspectos físico, psiquiátrico e social, assim como seus potenciais comportamentos de risco para a transmissão ou infecção pelo HIV. Todos os pacientes receberiam as instruções sobre o preenchimento destes fomulários, além de serem submetidos a procedimento padrão de pesquisa com consentimento informado. Os diferentes componentes relativos à aplicação de instrumentos e identificação de situações de risco podem ser acompanhados através da Figura 1. Os pacientes seriam atendidos em fluxo contínuo sem marcação de consulta, em um regime de atendimento rápido, desenvolvido e já utilizado pelo programa de dependência química do Hospital de Clínicas de Porto Alegre (HCPA). O programa previa um máximo de aquisição de quatro novos grupos de pacientes por mês. As consultas teriam um tempo médio de 90 minutos e os paciente seriam testados sistematicamente para o HIV. A freqüência proposta às consultas seria semanal nos dois primeiros meses do projeto, passando após para atendimentos bimestrais. Um supervisor de campo e um de atendimento seriam responsáveis pela captação e atendimentos dos pacientes, servindo de elo de ligação com as comunidades envolvidas no projeto. As atividades de grupo iriam utilizar as técnicas de abordagem de rede social descritas por Latkin et al. (1995) e Mandell et al. (1994), traduzidas e adaptadas para a utilização em nosso meio. Esta metodologia consiste de sessões de grupo utilizando o paciente-chave como ponto de referência para a formação progressiva da rede no ambiente do POAVHIVE. A cada sessão o terapeuta encorajaria o paciente-chave através de técnicas motivacionais a manter a sua rede social, desenvolvendo técnicas de negociação para sexo seguro e não compartilhamento de seringas, em parte baseadas na experiência já existente nos Centros de Orientação e Apoio Sorológico (COAS) de Porto Alegre, com os quais os autores do projeto já trabalhavam previamente. Seria realizado um inventário de necessidades sociais dos indivíduos e de cada rede social, utilizando um modelo social de uso de substâncias. Para cada indivíduo estava previsto o reforço dos laços com membros da família que estivessem disponíveis para participar das atividades do programa e auxiliar na recuperação do UDI. Este trabalho seria supervisionado sistematicamente por uma assistente social com treinamento específico na área. Também seriam realizados grupos focais em que se registraria o dis- 
Figura 1

Fluxograma de atendimento proposto

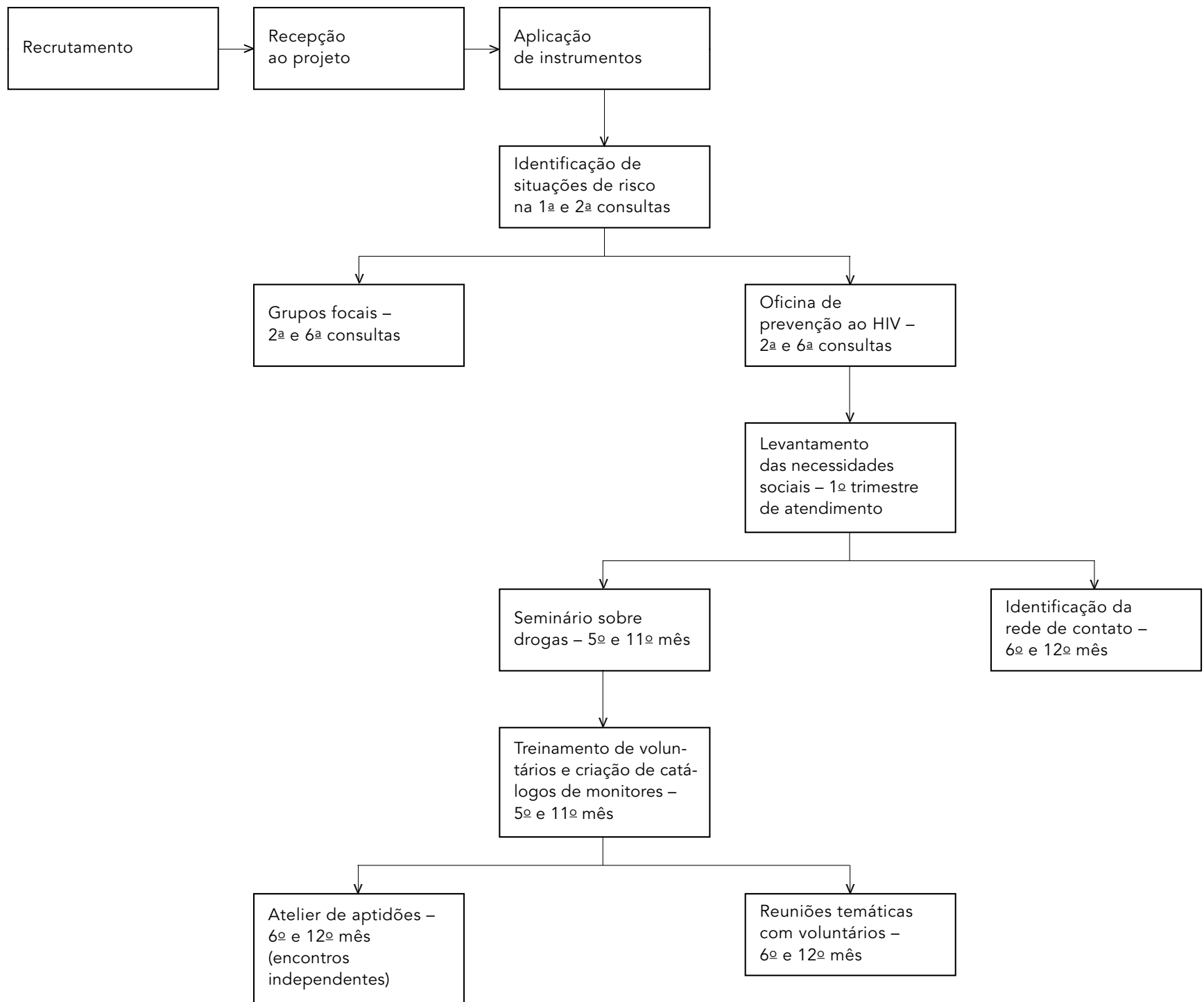

curso do paciente e de seu grupo para posterior análise de conteúdo. Quando necessário seriam utilizadas visitas in loco por assistentes sociais especificamente treinados. Nas atividades de desenvolvimento de aptidões específicas (Oficinas de Prevenção), seriam mantidas listas de profissionais treinadores em diversos ofícios que poderiam, sem ônus para o projeto, desenvolver diversos cursos com os pacientes. Ao final do projeto, agentes de saúde oriundos do programa seriam treinados para disseminar aspectos preventivos e didáticos para a comunidade.

\section{Equipe técnica}

A equipe técnica do POAVHIVE sofreu alterações ao longo da execução do projeto, o que certamente ilustra as mudanças de rota que se fizeram necessárias em sua trajetória. Ao final de um ano, a coordenação do POAVHIVE era composta por um psiquiatra sênior, especialista em dependência química, uma analista de dados, uma secretária, quatro psiquiatras juniores, três assistentes sociais, um consultor médico e uma consultora em Serviço Social. 
Inicialmente, o gerenciamento do trabalho era feito por uma enfermeira psiquiátrica, que após alguns meses desligou-se desta atividade, passando tal função a ser realizada pelo coordenador do projeto. Alguns terapeutas também se desligaram da atividade em seu início. À medida que a equipe foi se familiarizando com a proposta de trabalho, assim como integrando as diferentes maneiras de utilizar as técnicas, $o$ grupo não sofreu mais alterações. Os atendimentos clínicos eram realizados pelos psiquiatras, dos quais apenas um tinha treinamento prévio adequado para trabalhar com dependência química. Os demais estavam realizando seu treinamento in loco. As três assistentes sociais eram responsáveis por integrar aspectos sócio-familiares ao atendimento psicoterápico dos UDI, sendo este trabalho supervisionado pela consultora de serviço social.

\section{Estrutura física e relações do projeto com o HCPA}

O POAVHIVE foi o primeiro projeto pensado exclusivamente para o atendimento de UDI no HCPA, e as reflexões descritas abaixo ilustram a necessidade de desenvolvimento de uma cultura prévia para a realização de projetos de tal natureza.

O local destinado aos atendimentos do POAVHIVE foi o Ambulatório de Álcool e Drogas do HCPA, que se localiza no andar térreo, ligado a um corredor de passagem comum a várias áreas do hospital. Esta é uma zona de grande fluxo de pacientes das diversas especialidades, não dispondo assim de uma sala ou área de espera específica para esta atividade. Mesmo com a negociação com a direção do hospital quanto aos aspectos de gratuidade, cuidados éticos - como sigilo e privacidade - e conceitos relativos à redução de danos, a coordenação do hospital sugeriu que não se fizesse qualquer tipo de campanha de divulgação pública do POAVHIVE, temendo um aporte excessivo de pacientes ao serviço. Tal atitude, entretanto, dificultou o fluxo de pacientes ao POAVHIVE, e este aspecto merece uma especial discussão, uma vez que são muitas as variáveis que fazem com que um UDI consiga chegar a um local de atendimento, mesmo quando já lhe são extremamente facilitados os meios para tal. Este é um processo seqüencial e elaborado, que se inicia com a percepção da existência de um problema, e continua até o real encontro com um profissional de saúde. Considerando a caracterização da população atendida e sua dificuldade em chegar ao local de atendimento, um projeto "hospitalocêntrico" já teria dificuldades em sua origem. Segundo o relato dos pacientes, passar por uma série de postos de identificação, um labirinto de corredores, secretárias que atendem uma enorme quantidade de pessoas, e uma longa espera até $o$ atendimento, torna-se uma tarefa claramente insuportável. Na tentativa de solucionar este problema, pensou-se em desenvolver uma abordagem extra-muros. Com o auxílio de profissionais do projeto já treinadas em abordagens de Redução de Danos, fez-se tentativas de realizar atendimentos na casa ou em locais de reunião dos UDI com sua rede. Tal atitude funcionou a contento para os pacientes que já haviam se identificado no centro de tratamento ambulatorial e portanto já possuíam vínculos com as terapeutas. Porém, a saída das terapeutas para a comunidade, além de criar dificuldades logísticas, como falta de segurança e perda de tempo no deslocamento e na localização dos domicílios, não produziu o efeito desejado na captação de mais pacientes para o projeto.

\section{Encaminhamento dos casos e evolução do atendimento}

Os casos atendidos vinham encaminhados de um dos centros de referência da Prefeitura de Porto Alegre para HIV/AIDS, o COAS Paulo César Bonfim. Além disso, alguns encaminhamentos de UDI vieram diretamente de profissionais do Projeto de Redução de Danos da Prefeitura, por profissionais de saúde que conheciam o programa, ou por procura direta de atendimento no Ambulatório de Álcool e Drogas do HCPA. Os critérios de inclusão no programa compreendiam pacientes que tivessem rede social identificável e relativamente regular, fazendo uso de drogas EV, compartilhado ou não, não estando abstinentes há mais de um mês (sendo portanto usuários ativos). Após a triagem o paciente era encaminhado para uma das terapeutas que passava a acompanhá-lo sistematicamente. A rotina dos atendimentos consistia em estabelecer um vínculo terapêutico e avaliar o estágio de motivação em que o paciente se encontrava, de acordo com o modelo de Estágios de Mudança proposto por Prochaska \& Diclemente (1986), utilizando elementos motivacionais como a alavanca principal para o desenvolvimento de mudanças estruturadas na trajetória de uso de drogas de um dependente químico, conforme descrevem Miller \& Rollnick (1991). Latkin (1995:182), ao revisar a literatura referente à utilização de redes sociais 
sob forma preventiva, confirma que "a estratégia de utilizar sub-redes de compartilhamento de drogas, que ocorrem naturalmente, como um veículo para mudanças de comportamento é baseada em teorias de influência social, e que (...) normas e pressões de grupo são determinantes de práticas de injeção arriscadas e atividade sexual desprotegida", o que daria consistência a modelos de intervenção desta natureza.

Cada paciente, ou grupo de parceiros, era convidado a comparecer ao ambulatório do HCPA uma a duas vezes por semana para entrevistas conduzidas por uma das psiquiatras do projeto, que ficava, a partir daí, responsável pelo atendimento do paciente e de sua rede. Utilizou-se para a captação de pacientes um modelo de "amostragem por alvos" (targeted sampling) (Watters \& Biernacki, 1986; Inciardi et al., 1997) e "bola de neve" (snowballing, ou chain referral) (Inciardi, 1986). Estes modelos foram utilizados porque os pacientes não são o que se poderia chamar de uma população facilmente identificável em seu meio natural, sendo extremamente difícil a sua chegada a serviços de saúde, conforme já descrito anteriormente. A "amostragem por alvos" se caracteriza pela identificação inicial de um paciente-chave ("alvo") da temática em questão - freqüentemente o UDI que procura o centro ou que apresenta grande intensidade de sintomatologia, sendo por isto facilmente identificável. A partir deste indivíduo, busca-se esgotar a rede de círculos concêntricos de suas relações, com o objetivo de estender a intervenção a todos os sujeitos potencialmente envolvidos em seus relacionamentos pessoais. O objetivo aqui é o de esgotar as redes conhecidas, seja por um indivíduo de uma rede só, seja por vários de várias redes. Muitos sujeitos nos foram encaminhados assim, com o objetivo de esgotar a rede de conhecimento dos sujeitos-chave, e neste sentido estimulando a participação de indivíduos que não iriam buscar atendimento por iniciativa própria, portanto inacessíveis à sistemática passiva de amostragem por conveniência no seu formato convencional. Não se deve esquecer, entretanto, que apesar de todos os cuidados com o método descrito acima, o uso de drogas é sempre uma prática ilegal ou "para-social", o que faz com que qualquer abordagem sobre o uso de drogas - em particular as injetáveis - esteja sujeita a uma subapreciação e distorção das características in natura dos casos existentes (Sorensen et al., 1991). Muitos pacientes que não apresentavam rede social foram triados, porém encaminhados para outros programas de tratamento. Pacientes que à triagem demonstrassem outras motivações que não a de tratamento, também não foram incluídos no programa.

Após o estabelecimento da rede de contato, o programa propunha o desenvolvimento com os pacientes de uma listagem de situações de exposição e transmissão do HIV que se aplicasse à sua rotina de vida, através de drogas e/ou circunstâncias que envolvessem relacionamentos sexuais, com o objetivo de conhecer as características peculiares de funcionamento de uma rede de parceiros (Vide na Figura 1: Identificação da Rede de Contato). Um ponto teórico importante: mesmo que se estivesse trabalhando com as redes de indivíduos, e com a malha de interações existente naquela rede social, o objetivo final da intervenção sempre seria o de modificar as atitudes de cada indivíduo que pudessem refletir em uma melhora nas interações de grupo. Para tal, buscava-se desenvolver uma maior consciência crítica, associada a um maior conhecimento sobre o "patamar de risco" em que cada indivíduo se encontrava. Desta forma, o grupo de terapeutas ensinava formas menos prejudiciais de injeção (como por exemplo a assepsia prévia do local da picada) ou através de demonstrações práticas sobre técnica de limpeza de seringas potencialmente contaminadas através do uso de hipoclorito de sódio. No que compete à transmissão sexual, além dos ensinamentos básicos referentes ao uso de preservativos masculinos, com a utilização de próteses penianas, também era ensinado o uso e a função do preservativo feminino, com o intuito de aumentar a adesão a este método. As técnicas clássicas de Prevenção de Recaída (Marlatt \& Gordon, 1993) e os conceitos de Redução de Danos e elementos de Entrevista Motivacional eram sistematicamente utilizados pelas terapeutas. A abordagem contou também com um componente sócio-familiar, trazido para a equipe através do trabalho das assistentes sociais do projeto, e que é descrito em mais detalhes abaixo.

A intervenção realizada pelo Serviço Social dentro do POAVHIVE fundamentou-se no pressuposto teórico da Abordagem de Rede (Sluzki, 1997). Segundo Sluzki, a rede social inclui o conjunto de vínculos interpessoais do sujeito como a família, amigos, relações de trabalho, estudo, inserção comunitária e práticas sociais. Esta abordagem prevê colocar em movimento as forças, potencialidades e recursos dentro do sistema familiar que possam gerar suporte, satisfações e controle necessários para lidar com os estresses, problemas e doença (Hartman \& Laird, 1983), gerando uma compreensão dos processos de integração psicossocial, promoção do bem-estar, desenvolvimento da identi- 
dade, consolidação dos potenciais de mudança e reinserção social e pessoal na comunidade. Com a inclusão do serviço social, as necessidades foram levantadas e atendidas através de ações que envolviam entrevistas com familiares dos pacientes, no hospital ou em visitas domiciliares, contato com instituições e entidades, e identificando recursos comunitários a serem utilizados por pacientes e suas famílias. Observou-se que, contrariando a teoria inicialmente proposta, na maioria das vezes a rede familiar era a única com a qual realmente o paciente contava, e mesmo assim os familiares se descreviam como "em estado caótico", desgastados devido à patologia do paciente. A busca constante de abordagens mais interacionais e criativas gerou a elaboração de alguns instrumentos que suscitassem uma participação mais constante dos pacientes e suas redes. Vários elementos com esta característica foram criados, e uma breve descrição destes é feita a seguir.

\section{Elementos de facilitação das intervenções terapêuticas}

\section{- Vídeo sobre preservativo feminino}

Foi criado, em parceria com a Faculdade de Comunicação da UFRGS, um vídeo educativo sobre o Preservativo Feminino (Surratt et al., 1998). O vídeo mostra as circunstâncias que envolvem as decisões sobre utilizar ou não o preservativo convencional e o feminino, além de divulgar, através de uma inserção de caráter mais técnico, as formas de infecção conhecidas do vírus da AIDS. O mesmo foi desenvolvido como coadjuvante de uma intervenção preventiva com mulheres usuárias dos COAS que estivessem motivadas a experimentar este novo método contraceptivo e de prevenção das doenças sexualmente transmissíveis, e que poderiam se beneficiar de elementos motivacionais para sua tomada de decisão.

\section{- Jogos interativos}

A partir dos conceitos do modelo de Crenças em Saúde (Rosenstock, 1974), e utilizando a estrutura de estágios de mudança proposta por Prochaska \& DiClementi (1986), foram criados dois jogos de cartas com a finalidade específica de auxiliar os pacientes e suas redes a desenvolver habilidades para a utilização de práticas seguras para atividade sexual e, negociação com parceiros da rede visando prevenção de recaída quanto ao uso de drogas. Estes jogos são descritos brevemente abaixo:
"Sexo seguro"-É jogado com um baralho de cartas que descrevem situações de envolvimento sexual em diferentes níveis de risco para a transmissão do HIV. Participam seis pacientes mais o facilitador, em um tabuleiro com as alternativas: Sem Risco, Arriscado, Muito Arriscado. O objetivo do jogo é promover discussões sobre a transmissão do HIV através do contato sexual e maneiras de prevení-lo, utilizando uma estratégia lúdica. $\mathrm{O}$ facilitador deve ser um profissional treinado em HIV/AIDS e ter algum conhecimento prévio em aconselhamento para dependência química, uma vez que o jogo foi desenvolvido para usuários de drogas que se envolvem em comportamentos sexuais de alto risco.

"Céu e Inferno" - É jogado com 20 fichas de texto por um grupo de não mais do que cinco indivíduos mais o facilitador. Dez fichas são caracterizadas como "Céu" e dez como "Inferno”, exemplificando as cognições contrastantes e hipersaturadas que estão presentes no tratamento de abusadores de drogas. As fichas contêm expressões pessoais que contemplam os riscos e benefícios do uso de drogas em diferentes situações grupais e individuais. Para cada carta "inferno" existe ao menos uma carta "céu", mas freqüentemente há cinco ou seis cartas "sadias" parar cada carta "perigosa". O objetivo do jogo é o de gerar um grupo de respostas positivas, adequadas e potencialmente utilizáveis para cada uma das situações complexas que são apresentadas pelas cartas "inferno", permitindo desta forma aos pacientes o desenvolvimento de habilidades para prevenir recaídas. O papel do facilitador é o de promover a discussão e eliciar exemplos vívidos, personalizados, dos membros do grupo para cada situação. O facilitador deve ter um treinamento prévio em aconselhamento para dependência química, uma vez que esta é uma atividade tipicamente orientada para terapia.

\section{Avaliação e críticas ao POAVHIVE}

O relato deste projeto tem a finalidade de analisar a aplicabilidade de alguns conceitos utilizados, bem como sugerir alternativas às dificuldades encontradas.

Constatou-se que houve dificuldades em vários níveis do projeto, desde a fase de planejamento até o período de execução. O POAVHIVE em seu desenho original possuía uma proposta inovadora baseada em metodologias testadas em centros especializados em tratamento e pesquisa com UDI de países desenvolvidos, e com repercussões terapêuticas de grande porte no meio científico da área. 
Logo no início do trabalho houve mudanças substanciais na equipe devido à saída de alguns integrantes e à entrada de novos técnicos, que participariam em várias etapas do processo terapêutico: o projeto passou para uma segunda fase com a entrada de assistente sociais, sendo então reavaliados algumas condutas e objetivos do trabalho. Logo ficou evidente que a magnitude proposta no projeto era inviável, o que motivou uma diminuição do número de casos atendidos e de objetivos a serem atingidos. Também foram evidenciadas dificuldades inerentes à captação de pacientes e na implementação metodológica do trabalho, uma vez que muitos dos instrumentos do POAVHIVE eram simultaneamente criados e testados, gerando insegurança nos técnicos, que lidavam com métodos ainda em desenvolvimento. Algumas características estruturais e filosóficas do projeto também foram alteradas neste momento para permitir ainda alguma factibilidade ao trabalho proposto. As principais modificações foram as seguintes: (a) Manutenção da existência de uma rede social junto ao paciente, mas sem a obrigatoriedade de o paciente comparecer ao hospital trazendo sua rede em um primeiro momento; (b) a obrigatoriedade de trabalhar apenas com pacientes motivados ou motiváveis para a intervenção, excluindo portanto casos em que este atributo não estivesse explicitamente presente; (c) a potencial inclusão de usuários não injetáveis, mas de grande risco para exposição ao HIV, e que tivessem algum tipo de rede de suporte, aumentando assim o escopo do programa.

Como resultado destas modificações propostas, aos poucos foi se evidenciando que os pacientes que estavam sendo encaminhados não vinham acompanhados de suas redes de parceiros de uso, mas sim de seus familiares e cônjuges, que em alguns casos também usavam drogas. Outra observação importante da equipe foi a de que os pacientes que chegavam para atendimento estavam em situação muito grave de descontrole do uso de drogas, eventualmente apresentando risco de suicídio e de overdose. Como o HCPA não possui um programa de internação para dependentes químicos, estes foram sempre internados dentro da unidade psiquiátrica, sob a responsabilidade de um psiquiatra sênior especialista em dependência química. Além da ansiedade natural experimentada por nossos pacientes neste momento, os riscos de abandono de tratamento aumentavam nestas condições. A percepção dos autores é a de que a falta total de estrutura para atendimento de pacientes usuários de drogas - injetáveis ou não - em nosso meio
(Pechansky, 1994) é uma das causas pelas quais não houve seleção dos pacientes que nos eram enviados por outros centros ou profissionais de referência.

Outra questão importante a ser avaliada no projeto diz respeito ao número de pacientes e suas redes. São muitas as explicações que sugerem o motivo pelo qual somente 16 UDI concluíram sua participação, apesar de termos avaliado um número bem maior de casos. Uma das primeiras dificuldades encontradas foi a pouca aceitação dos pacientes em trazer a sua rede à terapia, dificuldade comum a todos os casos. Entendemos que a estrutura adequada para atender a população de UDI não é a que se insere dentro de um hospital, onde existe um policiamento presente dentro e fora da instituição, e uma sensação onipresente de que este tipo de paciente não é bem vindo. Também, o difícil acesso às salas de atendimento, a necessidade de identificar-se ao entrar no hospital, o fato de não existir um local específico dentro de um ambulatório com outras especialidades para o atendimento deste grupo de pacientes em especial, acabou estimulando ansiedades que constrangiam os participantes que eventualmente conseguiam chegar até o local de atendimento. Pensa-se que o indicado para esta população seja o modelo não hospitalar, como hospital-dia, hospital-turno ou Centros de Atenção Psicossocial . Esta estrutura existe noutros países, mas ainda é pouco habitual em nosso meio. O trabalho de rede como preconizado inicialmente acabou não tendo o êxito desejado, apesar dos resultados individuais terem sido bastante favoráveis aos pacientes que se envolveram no programa. Por parte dos terapeutas, é importante lembrar que o atendimento de redes sociais é prática incomum em nosso meio. As terapeutas envolvidas no projeto haviam recebido uma formação psiquiátrica voltada para o atendimento individual, no máximo incluindo familiares. A falta de um modelo já padronizado de atendimento pode ter sido outro fator limitante no andamento do trabalho. Acredita-se que a insegurança quanto ao método proposto, além do pouco treinamento dado aos terapeutas (apenas o coordenador do projeto havia sido treinado nesta técnica específica nos Estados Unidos) possam ter sido fatores decisivos para este desvio de rota. Outro fator a ser considerado para a pouca manutenção do atendimento das redes é o de que possivelmente o paciente-chave, que era inicialmente identificado para o projeto, estivesse em um estágio de motivação diferente do de seus parceiros, os quais não teriam então o mesmo interesse em comparecer 
ao centro de atendimento. Além disso, muitos dos UDI já tinham tido experiências prévias de tratamento e, certamente, em nenhuma destas foram incentivados a incluir sua rede para terapia. Mesmo assim, todos os elementos clínicos dos pacientes avaliados apontavam para uma significativa redução do número de drogas utilizadas, da quantidade de utilizações de cada droga, e em alguns casos a migração do uso injetável diário para um consumo ocasional, ou mesmo a interrupção total do uso de drogas por via endovenosa. O resultado, neste sentido, parece encorajador, apesar de não ser mensurável sob um formato estandardizado, para este tipo de paciente submetido a tamanho risco.

\section{Sugestões para projetos futuros}

É possível considerar um amadurecimento geral dos profissionais envolvidos neste tipo de atendimento, em particular dos psiquiatras, menos acostumados a abordagens generalizadas de pacientes em seu meio sócio-cultural, e predominantemente treinados para atendimentos individuais focados em sintomatologia clínica presente. Entretanto, é nossa vivência que o eventual desenvolvimento de novos projetos nesta área deveria acarretar um grande número de modificações, devendo obrigatoriamente: (a) Discutir e homogeneizar os conceitos e técnicas a serem empregados, no sentido de que todos os profissionais envolvidos estejam de acordo com os modelos de intervenção propostos. Como é frequente a presença de outra doença psiquiátrica associada ao uso de drogas, também é fundamental que o técnico tenha sido treinado no atendimento de comorbidades; (b) considerar a importância das questões geográficas e logísticas para o atendimento destes pacientes. É provável que o melhor local de abordagem desta população não seja um hospital ou mesmo qualquer ambiente médico, mas sim algum outro centro com características mais de atendimento comunitário, e com uma identidade mais próxima à do paciente; (c) diminuir o tempo de espera para atendimento, por fatores já previamente descritos. Progressivamente, após as primeiras vezes em que o paciente for recebido de forma quase imediata, sua capacidade de aguardar seu tempo de atendimento irá aumentar; (d) realizar estudos-piloto tanto no que compete à sistemática de captação de pacientes quanto à utilização de métodos inovadores; (e) certificar-se da firmeza das relações entre a equipe assistencial e os diversos focos de auxílio paralelo em projetos desta natureza: prefeitura, hospitais, centros comunitários, grupos voluntários de apoio; (f) inserir o projeto em uma malha de atendimento que inclui a opção de assistência ambulatorial, internação hospitalar total e parcial, e projetos similares a intervenções terapêuticas da magnitude de uma hospitalização em regime parcial (hospital-dia ou hospital-turno); (g) propiciar acesso facilitado a outros serviços médicos especializados, como infectologia, que devem manter estreita relação com o projeto, inclusive no que diz respeito aos pressupostos teóricos e filosóficos que embasam o atendimento.

\section{Agradecimentos}

Os autores agradecem à Dra. Alayde Pilla Barcellos e às assistentes sociais Maria Aparecida Grossini, Cintia da Silva Leal e Maria Regina Philomena, pela valiosa contribuição quando da confecção dos primeiros textos referentes a este artigo. Os autores também agradecem ao Ministério da Saúde, através do United Nations Drug Control Program, por ter financiado o Programa POAVHIVE - Venha Enfrentar o HIV. 


\section{Referências}

BARCELLOS, N., 1998. AIDS no Estado do Rio Grande do Sul-Avaliação Epidemiológica dos Casos Notificados até 31 de Março de 1998. Porto Alegre: Departamento de Ações em Saúde, Seção de Controle de AIDS, Secretaria da Saúde e do Meio Ambiente. (mimeo.)

HARTMAN, A. \& LAIRD, J., 1983. Family-Centered Social Work Practice. New York: The Free Press.

INCIARDI, J. A., 1986. The War on Drugs: Heroin, Cocaine, Crime, and Public Policy. Palo Alto: Mayfield Publishing.

INCIARDI, J. A.; SURRATT, H. L. \& McCOY, H. V., 1997. Establishing and HIV/AIDS intervention program for street drug users in a developing nation. Journal of Drug Issues, 27:173-193.

LATKIN, C., 1995. A personal network approach to AIDS prevention: An experimental peer group in SAFE study. In: Social Networks, Drug Abuse, and HIV Transmission (R. Needle, S. G. Genser, \& R. T. Trotter, eds.), pp. 181-195, NIDA Research Monograph 151, Rockville: National Institute on Drug Abuse.

LATKIN, C.; MANDELL, W.; VLAHOV, D.; KNOWLTON, A.; OZIEMKOWSKA, M. \& CELENTANO, D., 1995. Personal network characteristics as antecedents to neele-sharing and shooting gallery attendance. Social Networks, 17:219-228.

MANDELL, W.; VLAHOV, D.; COHN, S.; LATKIN, C. \& OZIEMKOWSKA, M., 1994. Correlates of needle sharing among intravenous drug users. American Journal of Public Health, 84:920-923.

MARLATT, G. A. \& GORDON, J. R., 1993. Prevenção de Recaída: Estratégias de Manutenção no Tratamento de Comportamentos Aditivos. Porto Alegre: Artes Médicas.

MILLER, W. R. \& ROLLNICK, S., 1991. Principles of motivational interviewing. In: Motivational Interviewing: Preparing People to Change Addictive Behavior (W. R. Miller \& S. Rollnick, eds.), pp. 51-63, London: Guilford Press.

PECHANSKY, F., 1994. Treatment for drug and alcohol problems in Brazil: A puzzle with missing pieces. Journal of Psychoactive Drugs, 27:117-123.
PECHANSKY, F.; INCIARDI, J. A. \& KOHLRAUSCH, E., 1996. Uma proposta para treinamento em intervenções preventivas sobre HIV/AIDS em Porto Alegre. Revista de Psiquiatria do Rio Grande do Sul, 18:374-371.

PECHANSKY, F.; SOIBELMAN, M. \& KOHLRAUSCH, E., 1994. Avaliação de Situações de Risco para a Transmissão do HIV em Porto Alegre, Brasil. Projeto de Pesquisa. Porto Alegre: Departamento de Psiquiatria, Universidade Federal do Rio Grande do Sul.

PECHANSKY, F.; SOIBELMAN, M. \& KOHLRAUSCH, E., 1997. Assessment of risk situations for HIV transmission among drug abusers in Porto Alegre, Brazil. Journal of Drug Issues, 27:147-154.

PROCHASKA, C. \& DiCLEMENTE, C., 1986. Toward a comprehensive model of change. In: Treating Addictive Behaviors: Processes of Change (W. Miller \& N. Heather, eds.), pp. 3-27, Nova York: Plenum.

ROSENSTOCK, I. M., 1974. Historical origins of the health belief model. Health Education Monographs, 2:328-335.

SANTOS, B.; BECK, E. J. \& PEIXOTO, M. F., 1994. Survival and medical intervention in southern Brazilian AIDS patients. International Journal of STD and AIDS, 5:279-283.

SLUZKI, C., 1997. A Rede Social na Prática Sistêmica. Alternativas Terapêuticas. São Paulo: Casa do Psicólogo.

SORENSEN, J. L.; WERMUTH, L. A.; GIBSON, D. R.; CHOI, K. H.; GUYDISH, J. R. \& BATKI, S. L., 1991. Preventing AIDS in Drug Users and Their Sexual Partners. New York: Guilford Press.

SURRATT, H. L.; WECHSBERG, W. M.; COTTLER, L. B.; LEUKEFELD, C. G.; KLEIN, H. \& DESMOND, D. P.,1998. Acceptability of the female condom among women at risk for HIV infection. American Behavioral Scientist, 41:1157-1170.

WATTERS, J. K. \& BIERNACKI, P., 1986.Targeted sampling: Options for the study of hidden populations. Social Problems, 36:416-430.

WOODY, G.; METZGER, D.; PECHANSKY, F. \& SOIBELMAN, M., 1995. A Disseminação do HIV entre Abusadores de Droga Brasileiros. Porto Alegre: National Institute on Drug Abuse. 